\title{
Dosimetric comparison of volumetric-modulated arc therapy and intensity-modulated radiation therapy in patients with cervical cancer: a meta-analysis
}

This article was published in the following Dove Press journal: OncoTargets and Therapy

Wei Bai'

\section{Changgui Kou' \\ Weiying $\mathrm{Yu}^{\prime}$ \\ Yuanyuan $\mathrm{Li}^{\prime}$ \\ Wanqing Hua' \\ Lei $\mathrm{Yu}^{2}$ \\ Jianfeng Wang ${ }^{3}$}

'Department of Epidemiology and Biostatistics, School of Public Health, Jilin University, Changchun I3002I, Jilin Province, China; ${ }^{2}$ Department of Radiotherapy, Second Hospital of Jilin University, Changchun I3004I, jilin Province, China; ${ }^{3}$ Department of Radiotherapy, China-Japan Union Hospital, Changchun I30033, Jilin Province, China
Correspondence: Lei Yu Department of Radiotherapy, 218 Ziqiang Street, Changchun I3004I, jilin Province, China

Tel +8643 I 8II3 6I79

Fax +86 43I 8II36179

Email yul0826@I63.com

Jianfeng Wang

Department of Radiotherapy,

China-Japan Union Hospital,

Jilin Province, China

Tel +8643184995438

Fax +86 43। 84995438

Email jfwang@jlu.edu.cn Second Hospital of Jilin University,

126 Xiantai Street, Changchun I30033,

Background: Intensity-modulated radiation therapy (IMRT) and volumetric-modulated arc therapy (VMAT) are two of the main treatment techniques for cervical cancer. Whether either technique significantly reduces irradiated volumes of organs at risk (OARs) remains controversial. The aim of this study was to explore which of these treatment paradigms is the superior technique in cervical treatment, taking clinical outcomes and treatment efficiency from published findings into consideration.

Materials and methods: PubMed, EMBASE, and Cochrane Library databases were utilized. The average percent irradiated volumes of OAR were extracted from all included studies. Dual arc results were extracted due to their superiority to single arc methods in terms of plan quality. Standard mean deviations and 95\% CIs were calculated for delivery time, monitor units, and average percent irradiated volumes of OAR. Assessment of publication bias and sensitivity analyses were performed. All statistical analyses were conducted using R 3.5.0 software.

Results: Eight studies were included in this meta-analysis. For irradiated volumes of OARs, irradiated volume of rectum receiving $40 \mathrm{~Gy}$ (rectum V40) was significantly decreased in VMAT compared with IMRT. However, no significant differences were observed between IMRT and VMAT plans in bladder V40 or small bowel V40/V30. In addition, delivery times and monitor units were significantly lower in the VMAT plan than in the IMRT plan.

Conclusion: Compared with IMRT, VMAT is significantly more protective for the rectum, suggesting that it may be an optional therapy technique for patients with cervical cancer.

Keywords: VMAT, IMRT, cervical cancer, meta-analysis

\section{Introduction}

Cervical cancer is the fourth most common type of cancer in women after breast, colorectal, and lung cancer and the seventh most common cancer overall. ${ }^{1}$ Radiotherapy plays a crucial role in the treatment of cervical cancer. Three-dimensional conformal radiation therapy is the most widely used treatment plan; however, it results in a large volume of surrounding healthy tissues being irradiated, including the rectum, bladder, small bowel, femoral heads, and other organs. According to multiple studies, ${ }^{2-6}$ intensity-modulated radiation therapy (IMRT) appears to reduce the radiation exposure to organs at risk (OARs) compared with the three-dimensional conformal radiation therapy technique for the treatment of various cancers.

Although there are significant benefits of using IMRT, disadvantages also exist. IMRT requires multiple fixed-angle beams, and it may result in longer treatment delivery

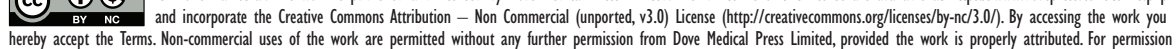
hereby accept the Terms. Non-commercial uses of the work are permitted without any further permission from Dove Medict
for commercial use of this work, please see paragraphs 4.2 and 5 of our Terms (https://www.dovepress.com/terms.php). 
time and greater discomfort in patients. ${ }^{7}$ Furthermore, a large number of monitor units (MUs) are applied during IMRT, which may increase low-dose radiation received by surrounding tissues, posing a greater risk for secondary radiationinduced malignancies. ${ }^{8}$

Volumetric-modulated arc therapy (VMAT), a new form of IMRT, provides a dynamic multileaf collimator, one gantry rotation, variable dose rate, and gantry speed. ${ }^{9}$ Several studies have reported that compared with conventional IMRT, VMAT conveys significant dose reduction to OARs, ${ }^{10-12}$ fewer MUs, ${ }^{2,10-12}$ and enhanced plan quality. ${ }^{13}$ Jia et $\mathrm{al}^{14}$ and Zhai et $\mathrm{al}^{15}$ found that there were no significant differences in the volume of irradiated OARs (small bowel, rectum, and bladder) between IMRT and VMAT plans, whereas Cozzi et $\mathrm{al}^{10}$ found that irradiated volumes of the rectum, bladder, and small bowel were decreased statistically significantly in VMAT plans. Sharfo et $\mathrm{al}^{13}$ found that compared with single arc, dual arc VMAT planning resulted in reduced OAR dose delivery. Due to differential design methods and small sample sizes, results from these studies are inconsistent.

Therefore, we performed a meta-analysis to explore which technique, IMRT or VMAT, is superior for the treatment of cervical cancer and to provide clinical guidance for choosing a suitable technique.

\section{Materials and methods}

\section{Search strategy}

PubMed, EMBASE, and Cochrane Library databases were searched by two authors (WB, WY) with no restrictions on years or status of publication. Language of publications was limited to English. The date of the last search was May 2018. The following keywords were used: "Uterine cervical neoplasms," "intensity modulated radiotherapy," "IMRT," "volumetric modulated arc radiotherapy," "VMAT," and "Rapid arc." The following combinations of the keywords were used: ([Uterine cervical neoplasms] AND [intensity modulated radiotherapy OR IMRT]) AND (volumetric modulated arc radiotherapy OR VMAT OR rapid arc). Retrieval strategies were adjusted accordingly for different databases and were confirmed many times after preretrieval tests of the combination of subject words and free words. To collect additional eligible studies, we also entered the keywords into the Google Scholar search engine.

\section{Selection criteria}

Two independent reviewers (WB, YL) selected and assessed data based on the following criteria: 1) Patients included in our analysis must be diagnosed with cervical cancer; 2) Studies describing IMRT and VMAT plans were included unless they were combined with other treatments, at which point they were not considered. Target volumes with simultaneous integrated boost were excluded; 3) Studies were included if they provided any of the following information: MUs, delivery time, dose-volume histograms to rectum, bladder, and small bowel; and 4) Case reports, conference abstracts, review articles, and studies regarding recurrences were excluded.

\section{Data extraction}

Data extraction was performed independently by two reviewers (WB, WH). Disagreements were solved by discussion and if necessary, another reviewer participated to reach a consensus. The following data were extracted from the included studies: 1) Basic characteristics, including first author's last name, publication year, country, title, sample size, and prescribed dose of IMRT and VMAT and 2) Data for delivery time, MUs, average percent irradiated volumes of OAR (rectum, bladder, and small bowel), and SD at various radiation doses in dose-volume histograms. These doses were selected based on information included in all studies reported, and all doses mentioned in the articles were extracted.

The detailed principle was as follows: if the prescribed dose in the study was $50 \mathrm{~Gy}$, the radiation doses of different organs were under $50 \mathrm{~Gy}$, so we only extracted data for radiation doses $<50 \mathrm{~Gy}$. If the article provided data for both single arc VMAT and dual arc VMAT, we only utilized dual arc results because it has been shown that dual arc VMAT is superior to single arc VMAT due to its superior plan quality. ${ }^{13}$

\section{Statistical analysis}

Measurement data using standardized mean difference (SMD) and $95 \%$ CIs were calculated. The SMD of irradiated volumes of the rectum, bladder, and small bowel at various radiation doses (30 and 40 Gy), delivery times, and MUs for IMRT and VMAT was compared. $I^{2}$ was used to test heterogeneity between studies. If heterogeneity was not present $\left(I^{2}<50 \%\right)$, a fixed effect model was applied for analysis; otherwise, a random effect model was adopted. Funnel plots and Begg's rank correlation test were used to assess potential publication bias. Sensitivity analysis was performed to assess the stability of the meta-analysis results. All statistical analyses were performed using R 3.5.0 software (www.r-project.org). $P<0.05$ was considered statistically significant.

\section{Results}

\section{Literature search}

A total of 976 articles were retrieved by the electronic search after exclusion of duplications. Ultimately, eight full-text 


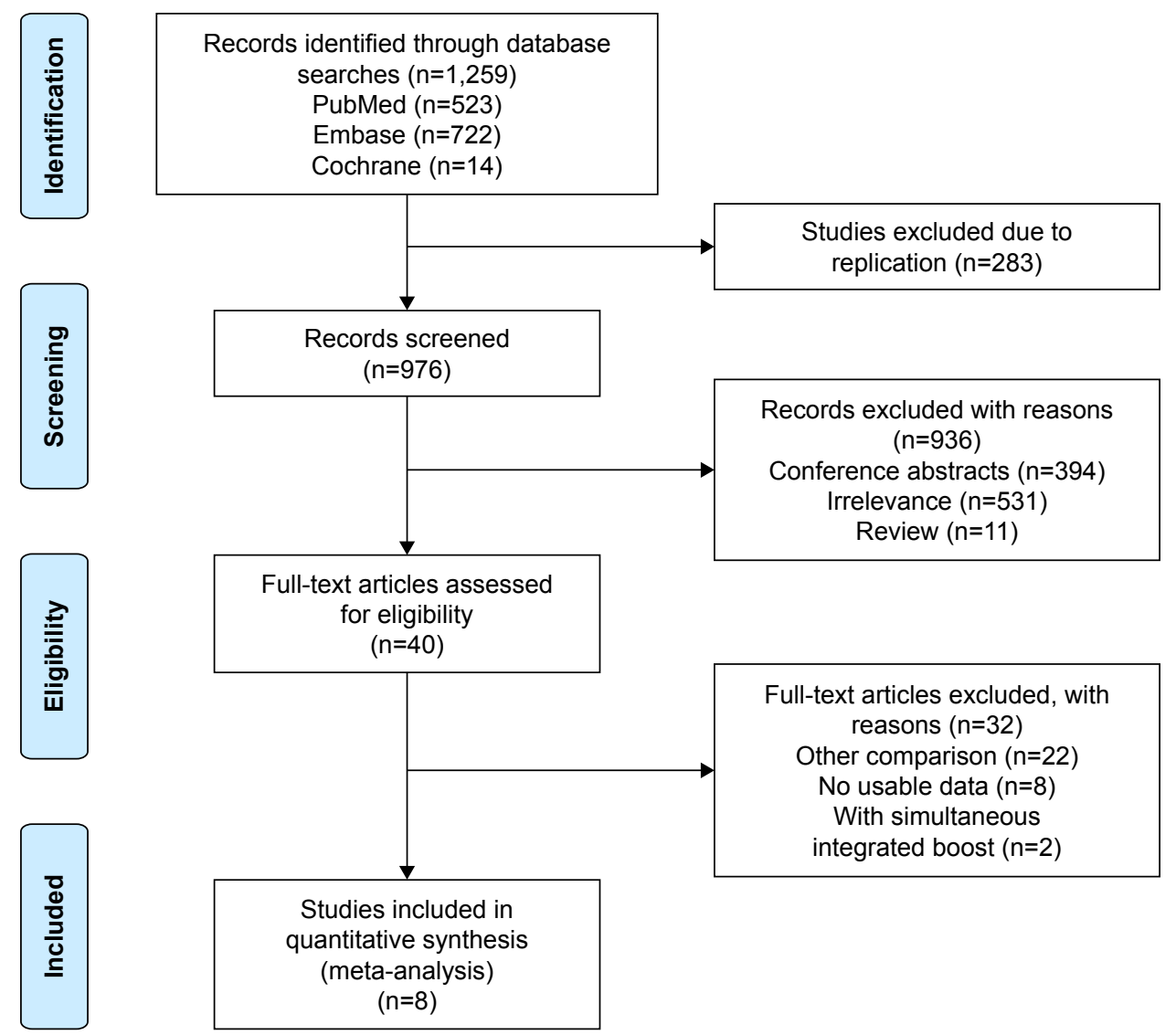

Figure I Flow diagram of retrieved studies.

articles were included in this meta-analysis after screening articles according to inclusion/exclusion criteria. A PRISMA flow diagram of studies is shown in Figure 1.

\section{Basic characteristics}

Basic characteristics, including the first author's last name, publication year, country, sample size, and other information, are summarized in Table 1. Three included studies ${ }^{7,11,17}$ reported data on postoperative cases, two studies ${ }^{11,17}$ reported that patients received postoperative adjuvant concurrent radiochemotherapy, and one study ${ }^{12}$ reported that patients received radical concurrent radiochemotherapy.

\section{Comparison of IMRT and VMAT}

For the dose to OAR, forest plots are shown only for rectum V40, bladder V40, and small bowel V40/V30 because studies containing rectum and bladder V30 were few in number (less than four studies). Forest plots show that rectum V40 $(\mathrm{SMD}=-0.27,95 \% \mathrm{CI}=-0.49,-0.05)$ was decreased in the VMAT plan compared with the IMRT plan (Figure 2).

Table I Basic characteristics of included studies

\begin{tabular}{|c|c|c|c|c|c|c|c|c|c|c|}
\hline \multirow[t]{2}{*}{ Year } & \multirow{2}{*}{$\begin{array}{l}\text { First author's } \\
\text { last name }\end{array}$} & \multirow[t]{2}{*}{ Country } & \multirow{2}{*}{$\begin{array}{l}\text { Prescribed } \\
\text { dose }\end{array}$} & \multicolumn{2}{|c|}{ Sample size } & \multicolumn{3}{|c|}{ OAR (level of the dose) } & \multirow[t]{2}{*}{ MUs } & \multirow{2}{*}{$\begin{array}{l}\text { Delivery } \\
\text { time }\end{array}$} \\
\hline & & & & IMRT & VMAT & Rectum & Bladder & Small bowel & & \\
\hline 2008 & Cozzi & Switzerland & 52.5 & 8 & 8 & V40 & V40 & V40 & Yes & No \\
\hline 2013 & Zhai & China & 56 & 12 & 12 & V30, V40 & V30, V40 & V30, V40 & Yes & Yes \\
\hline 2014 & Jia & China & 50 & 9 & 9 & V40 & V40 & V40 & Yes & Yes \\
\hline 2016 & Qiao & China & 50 & 15 & 15 & V30, V40 & V30, V40 & V30, V40 & Yes & Yes \\
\hline 2016 & Guy & France & 50 & 10 & 10 & V40 & V40 & V40 & Yes & Yes \\
\hline 2017 & Deng & China & 45 & 15 & 15 & V40 & V40 & V30, V40 & Yes & Yes \\
\hline 2018 & Yoshihiro & Japan & 50.4 & II & II & V40 & V40 & V40 & No & No \\
\hline 2018 & Guo & China & 45 & 84 & 84 & V30, V40 & V30, V40 & V30, V40 & Yes & Yes \\
\hline
\end{tabular}

Note: The dose to the OAR is presented as the percentage volume $(\mathrm{V})$ of the organ receiving $\mathrm{n}$ dose in gray $(\mathrm{Vn})$.

Abbreviations: IMRT, intensity-modulated radiation therapy; MU, monitor unit; OAR, organs at risk; VMAT, volumetric-modulated arc therapy. 


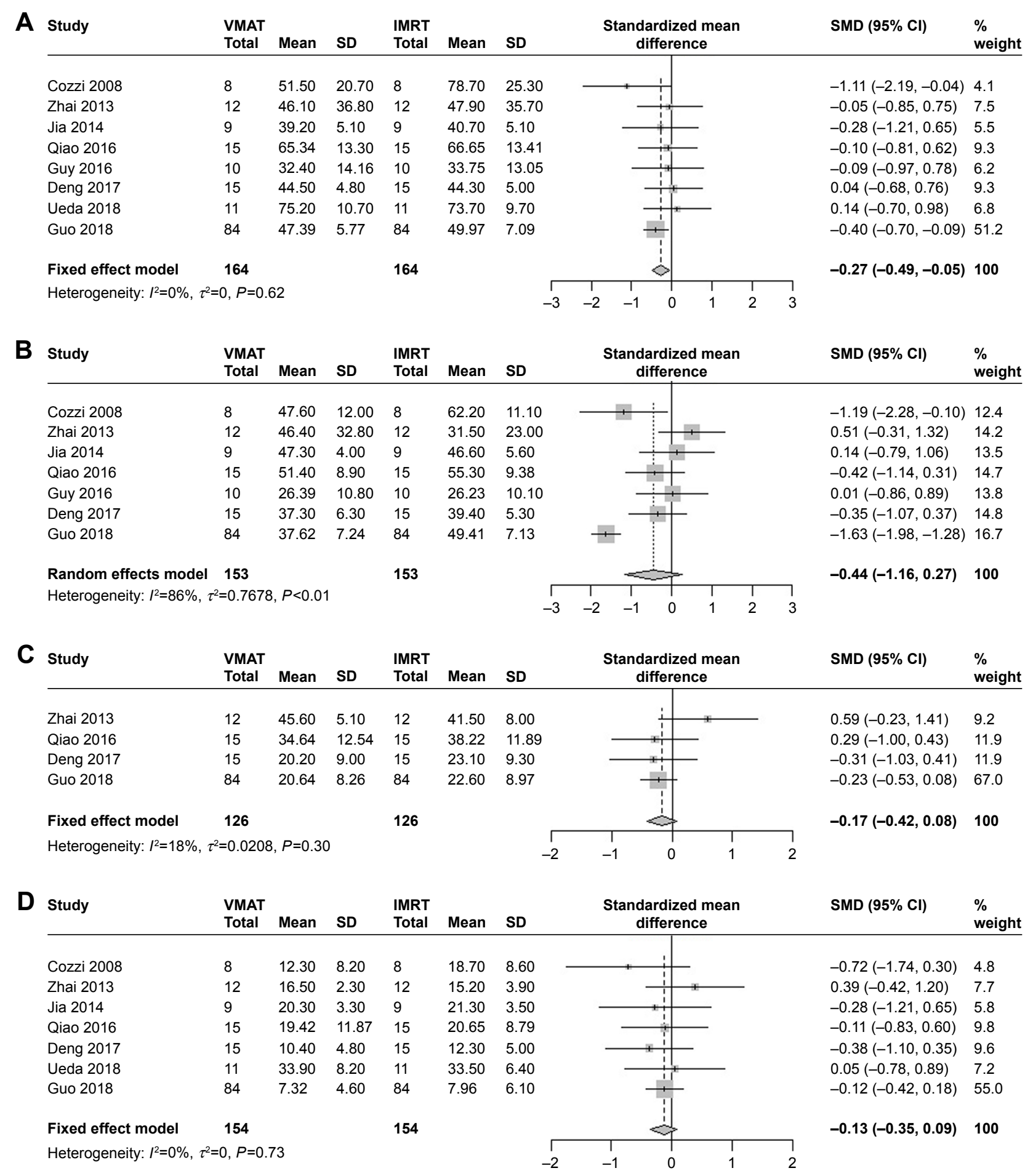

Figure 2 Comparison of irradiated volumes of OARs between IMRT and VMAT.

Notes: (A) Rectum V40; (B) Bladder V40; (C) Small bowel V30; (D) Small bowel V40.

Abbreviations: IMRT, intensity-modulated radiation therapy; OAR, organs at risk; VMAT, volumetric-modulated arc therapy.

However, there were no statistical differences between the IMRT and VMAT plans in bladder V40 or small bowel V40/ V30 (Figure 2). In addition, delivery time ( $\mathrm{SMD}=-10.11$, 95\% $\mathrm{CI}=-14.26,-5.96)$ and MUs (SMD $=-9.52,95 \% \mathrm{CI}=-$ $14.35,-4.69)$ were significantly lower in the VMAT plan than in the IMRT plan (Figure 3).

\section{Publication bias and sensitivity analysis}

Funnel plots for irradiated volumes of different organs, delivery time, and MUs are shown in Figure 4. Included studies in the funnel plots were symmetrically distributed, and Begg's test was employed to assess publication bias, with all the $P$-values $>0.05$. There was no publication bias 


\section{Delivery time}

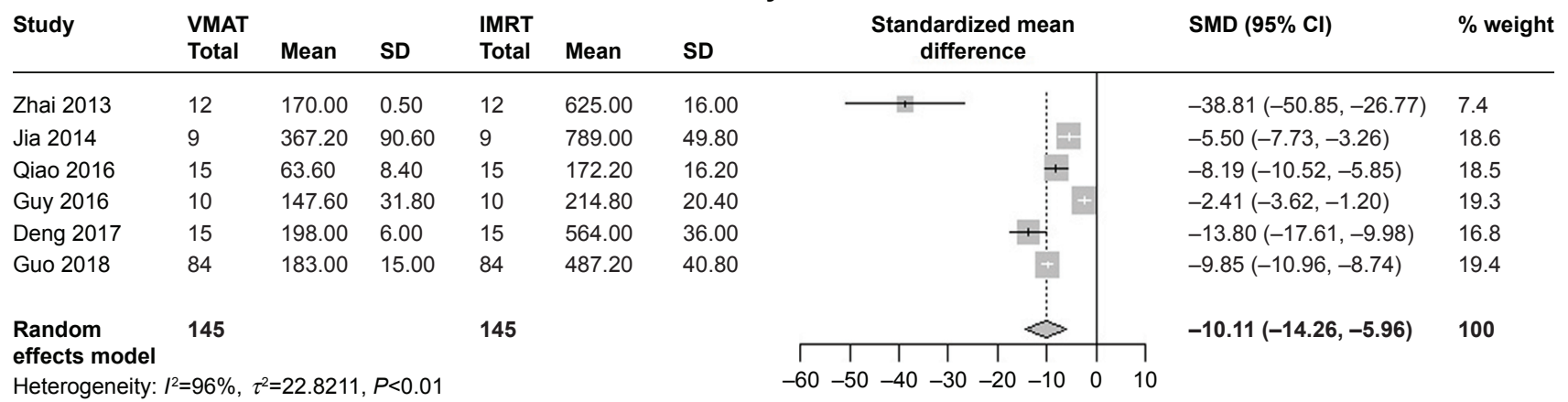

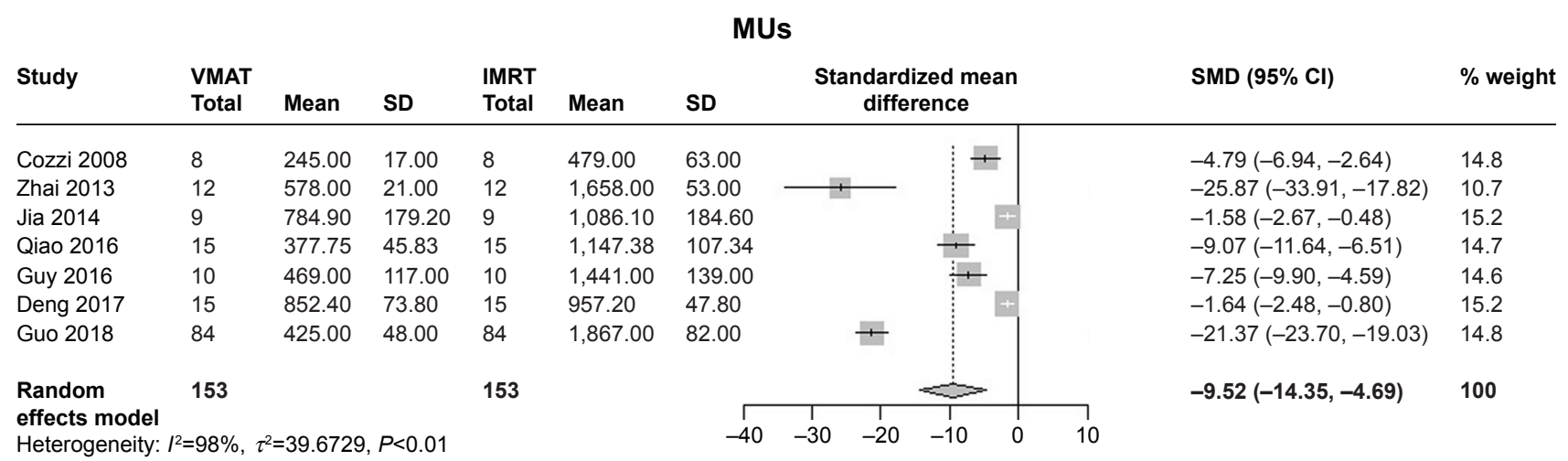

Figure 3 Comparison of the treatment efficiency between IMRT and VMAT.

Abbreviations: IMRT, intensity-modulated radiation therapy; MUs, monitor units; OAR, organs at risk; VMAT, volumetric-modulated arc therapy.

for any of these indicators. In addition, sensitivity analyses were conducted, and the results are shown in Figure 5. We found that only the results for rectum V40 (Figure 5A) may not be stable.

\section{Discussion}

To the best of our knowledge, this is the first meta-analysis combining multicenter results to explore dosimetric differences between VMAT and IMRT plans for treating cervical cancer. Our results showed that the VMAT plan reduces the volume of irradiated rectum and provides better treatment efficiency.

The rectum is an important OAR in cervical cancer. Cozzi et al, ${ }^{10}$ Qiao et al, ${ }^{11}$ and Guo et $\mathrm{al}^{12}$ reported that the V40 was lower in VMAT compared with IMRT, whereas other included studies found that there were no significant reductions in average percent volume of rectum irradiated at $40 \mathrm{~Gy}$ between the two plans. Although sensitivity analysis in our study indicated that rectum V40 results (Figure 5) may not be stable due to the study conducted by Guo et al, ${ }^{12}$ we maintain that compared with IMRT, VMAT is much more protective for the rectum because sample size of the study conducted by Guo et $\mathrm{al}^{12}$ was large enough. These inconsistent results may arise from small sample sizes and plan methods. Our meta-analysis indicated that rectum V40 $(\mathrm{SMD}=-0.27,95 \%$
$\mathrm{CI}=-0.49,-0.05)$ was significantly decreased in the VMAT plan. Similar results were obtained among prostate cancer patients. ${ }^{16}$ A meta-analysis ${ }^{16}$ reported that compared with the IMRT plan, the VMAT plan reduced the volume of irradiated rectum at doses of 40, 60, and 70 Gy for the treatment of prostate cancer. For the rectum, our study did not analyze irradiation doses lower than 40 Gy because the number of articles with these data was fewer than four.

Another important organ that should be protected in radiotherapy is the bladder. There were no significant dosimetric differences observed in the bladder, which was also reported in the treatment of prostate cancer at various doses by Ren et al, ${ }^{16}$ and heterogeneity existed between included studies in our meta-analysis $\left(I^{2}=86 \%, P<0.01\right)$ due to small sample sizes and plan strategies. These results indicate that the radiation volume of this organ at different doses may not be primarily influenced by different techniques but may be a result of multiple factors, such as algorithm and beam angles. ${ }^{16}$

For the small bowel, we analyzed different doses (40 and $30 \mathrm{~Gy}$ ) to compare dosimetric differences between IMRT and VMAT plans. Zhai et $\mathrm{al}^{15}$ found that IMRT was superior to VMAT, whereas other included studies found no differences. After combining multicenter data, our meta-analysis showed that there were no significant differences between these two plans for this organ. We did not analyze irradiation doses 


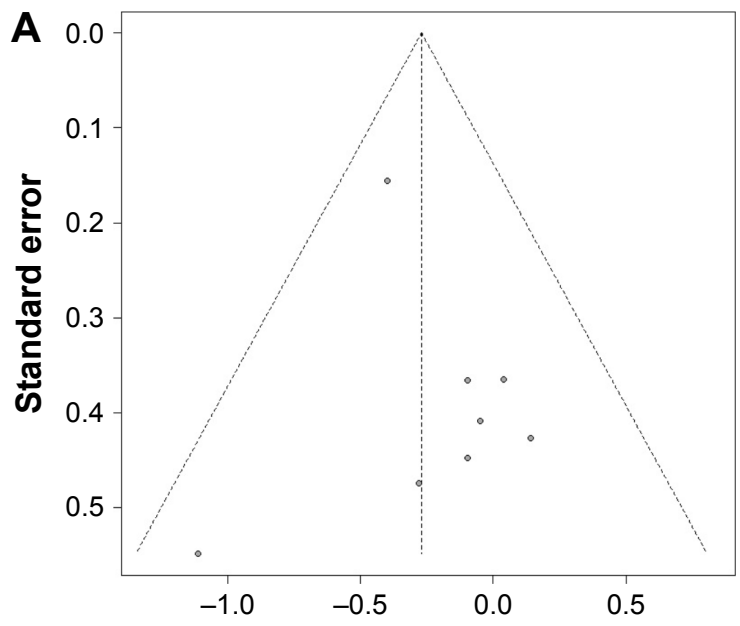

Standardized mean difference
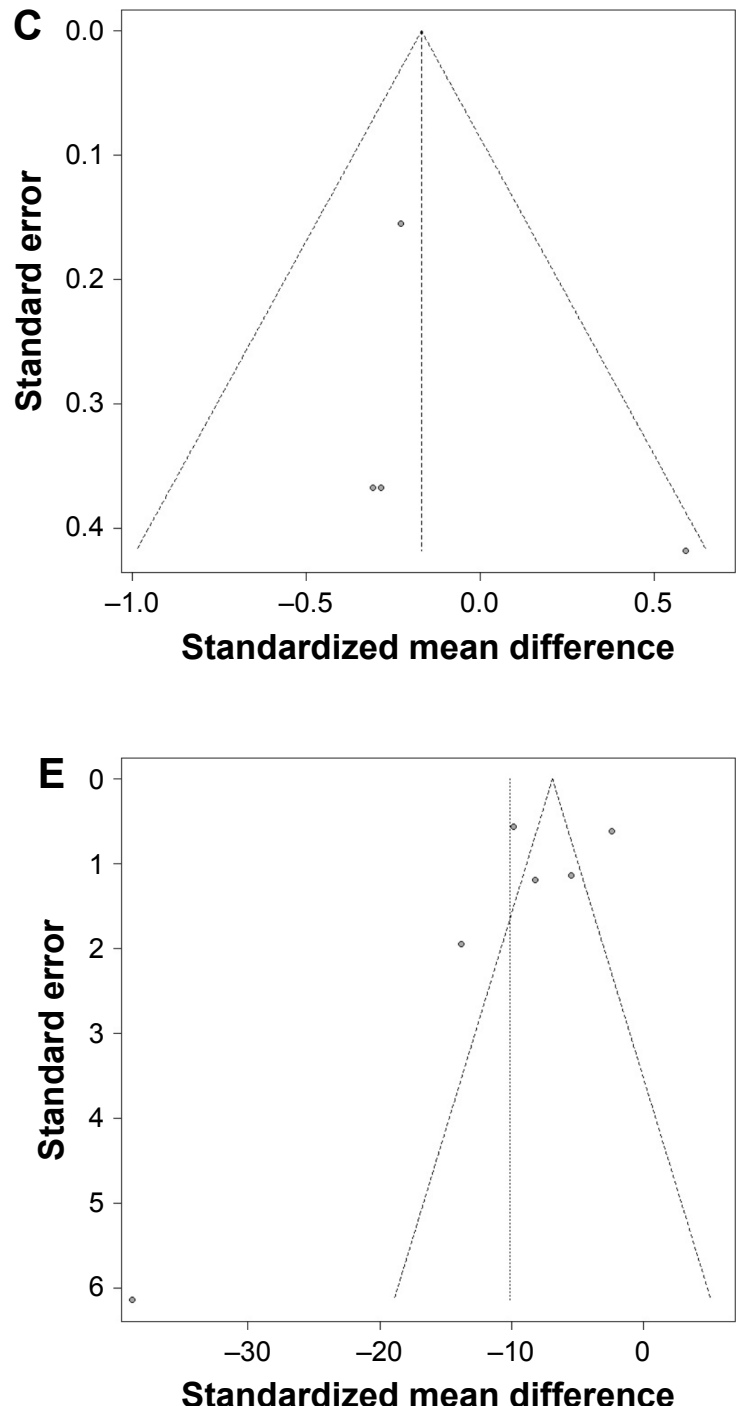
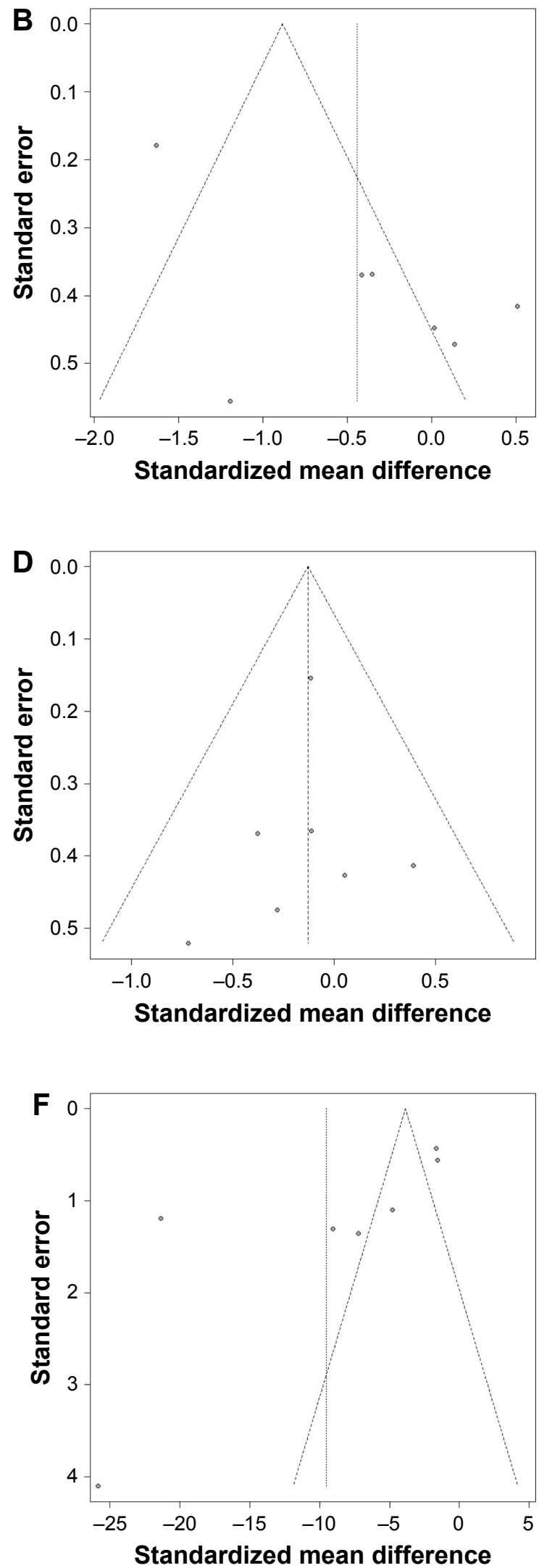

Figure 4 Funnel plots of irradiated volumes of OARs, delivery time, and MUs.

Notes: (A) Rectum V40; (B) Bladder V40; (C) Small bowel V30; (D) Small bowel V40; (E) delivery time; (F) MUs.

Abbreviations: MUs, monitor units; OAR, organs at risk. 


\section{A}

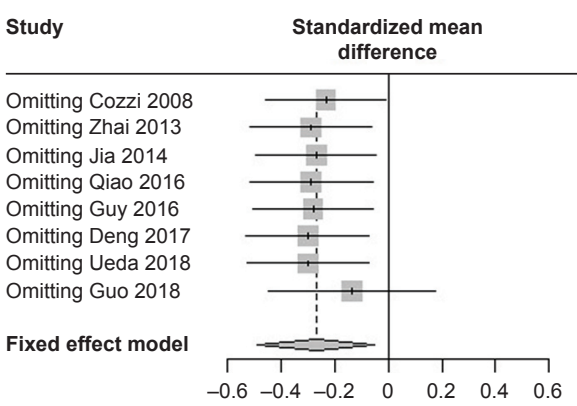

B

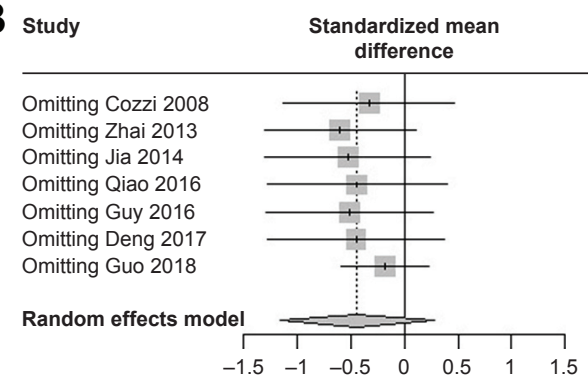

C

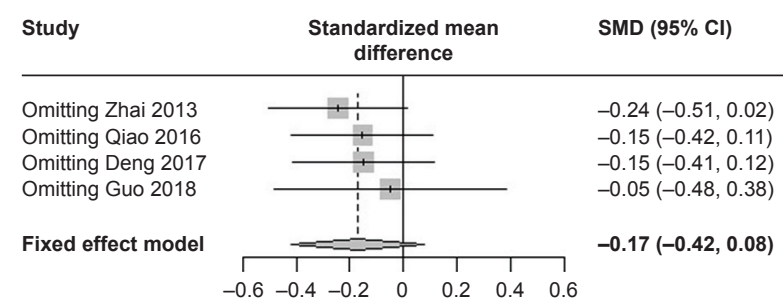

D

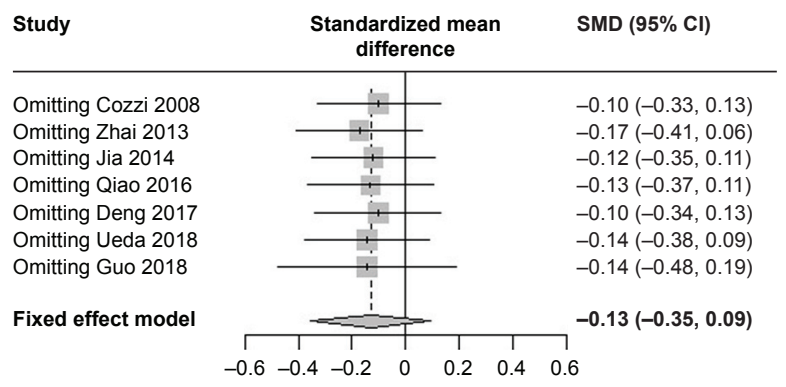

E

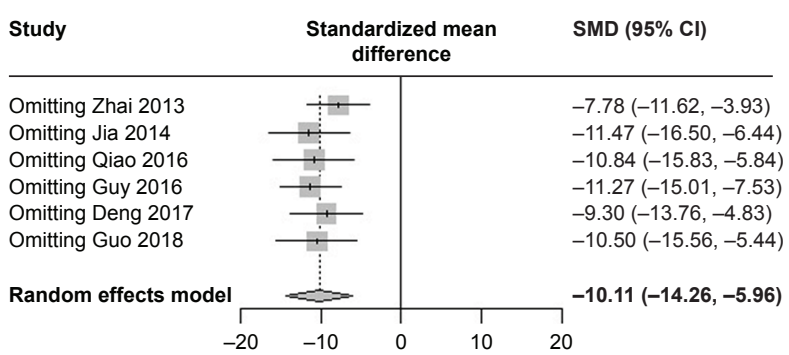

F study

Standardized mean $\quad$ SMD $(95 \% \mathrm{Cl})$ difference

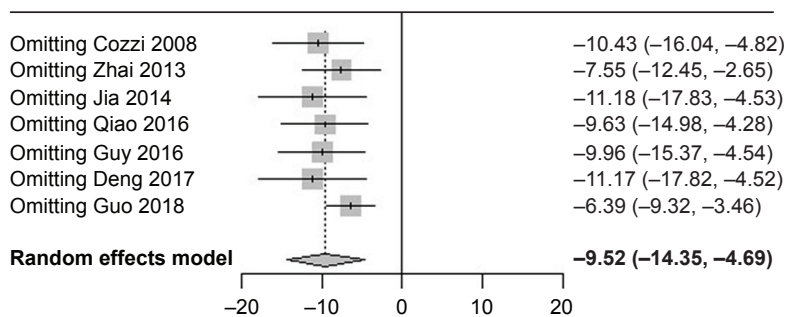

Figure 5 Sensitivity analyses of irradiated volumes of OARs, delivery time, and MUs.

Notes: (A) Rectum V40; (B) Bladder V40; (C) Small bowel V30; (D) Small bowel V40; (E) delivery time; (F) MUs.

Abbreviations: MUs, monitor units; OAR, organs at risk; SMD, standardized mean difference.

lower than 30 Gy because only three articles ${ }^{11,12,15}$ contained V10 and V20 data.

This study also analyzed the treatment efficiency of the IMRT and VMAT plans. Our meta-analysis indicated that MUs (SMD $=-9.52,95 \% \mathrm{CI}=-14.35,-4.69)$ were significantly reduced in the VMAT plan. Because there were fewer MUs in the VMAT plan, it was clear that delivery time ( $\mathrm{SMD}=-10.11,95 \% \mathrm{CI}=-14.26$ to -5.96$)$ was shorter in the VMAT plan than in the IMRT plan. Heterogeneous results were observed in MUs and delivery time due to differential planning strategies and algorithms, which were similar to results reported by Ren et al. ${ }^{16}$ Despite this issue, we can still conclude that VMAT provides better delivery efficiency and conclude that this method is the superior recommended method of treatment.

There were several limitations in this meta-analysis. First, included studies were observational, which may not provide accurate findings; however, after synthesizing the results of multicenter studies with small sample sizes, our meta-analysis can still provide some value for comparing the advantages between these two radiotherapy techniques. Second, there were heterogeneous results in our meta-analysis due to small sample sizes and inevitable clinical heterogeneity, such as incomplete information of disease status, different stages of cervical cancer and gross tumor volume sizes, and different interventions, such as planning strategies, optimization algorithms, contouring quality, and geometric expansion distances to planning target volume. Sensitivity analysis revealed that the results of irradiated volumes to the rectum were not stable. Unfortunately, only one included study ${ }^{12}$ in this meta-analysis listed data for acute and chronic complications, making it difficult to assess individual patient data.

Consequently, to obtain more precise results and to improve clinical decisions, studies with larger sample sizes 
and studies with sufficient documentation of the variety of clinical heterogeneity using contouring methods with consensus and describing the toxicity of the two techniques with longer follow-up are required.

\section{Conclusion}

Compared with IMRT, VMAT is significantly more protective for the rectum. Hence, VMAT may be an optional therapeutic technique for cervical cancer.

\section{Author contributions}

LY, JW, WB, and CK contributed substantially to conception and design; WB, WY, YL, and WH to acquisition of data; or WB, CK, LY, and JW to analysis and interpretation of data. WB, CK, WY, YL, and WH drafted the article or LY and JW revised it critically for important intellectual content. All authors finally approved the version to be published. All authors agreed to be accountable for all aspects of the work in ensuring that questions related to the accuracy or integrity of any part of the work are appropriately investigated and resolved.

\section{Disclosure}

The authors report no conflicts of interest in this work.

\section{References}

1. Ferlay J, Soerjomataram I, Dikshit R, et al. Cancer incidence and mortality worldwide: sources, methods and major patterns in GLOBOCAN 2012. Int J Cancer. 2015;136(5):E359-E386.

2. Guy JB, Falk AT, Auberdiac P, et al. Dosimetric study of volumetric arc modulation with RapidArc and intensity-modulated radiotherapy in patients with cervical cancer and comparison with 3-dimensional conformal technique for definitive radiotherapy in patients with cervical cancer. Med Dosim. 2016;41(1):9-14.

3. Wang X, Li G, Zhang Y, et al. Single-arc volumetric-modulated arc therapy (sVMAT) as adjuvant treatment for gastric cancer: dosimetric comparisons with three-dimensional conformal radiotherapy (3D-CRT) and intensity-modulated radiotherapy (IMRT). Med Dosim. 2013;38(4):395-400.

4. Shen Q, Ma X, Hu W, Chen L, Huang J, Guo Y. Intensity-modulated radiotherapy versus three-dimensional conformal radiotherapy for stage I-II natural killer/T-cell lymphoma nasal type: dosimetric and clinical results. Radiat Oncol. 2013;8:152.
5. Nour AA, Alaradi A, Mohamed A, Altuwaijri S, Rudat V. Intensity modulated radiotherapy of upper abdominal malignancies: dosimetric comparison with 3D conformal radiotherapy and acute toxicity. Radiat Oncol. 2013;8:207.

6. Yang B, Zhu L, Cheng H, Li Q, Zhang Y, Zhao Y. Dosimetric comparison of intensity modulated radiotherapy and three-dimensional conformal radiotherapy in patients with gynecologic malignancies: a systematic review and meta-analysis. Radiat Oncol. 2012;7:197.

7. Deng X, Han C, Chen S, et al. Dosimetric benefits of intensity-modulated radiotherapy and volumetric-modulated arc therapy in the treatment of postoperative cervical cancer patients. J Appl Clin Med Phys. 2017; 18(1):25-31.

8. Ruben JD, Davis S, Evans C, et al. The effect of intensity-modulated radiotherapy on radiation-induced second malignancies. Int J Radiat Oncol Biol Phys. 2008;70(5):1530-1536.

9. Otto K. Volumetric modulated arc therapy: IMRT in a single gantry arc. Med Phys. 2008;35(1):310-317.

10. Cozzi L, Dinshaw KA, Shrivastava SK, et al. A treatment planning study comparing volumetric arc modulation with RapidArc and fixed field IMRT for cervix uteri radiotherapy. Radiother Oncol. 2008;89(2): 180-191.

11. Qiao L, Cheng J, Liang N, Xie J, Luo H, Zhang J. A comparative dosimetric study of volumetric-modulated arc therapy vs. fixed field intensity-modulated radiotherapy in postoperative irradiation of stage IB-IIA high-risk cervical cancer. Oncol Lett. 2016;11(2): 959-964.

12. Guo M, Huang E, Liu X, Tang Y. Volumetric modulated arc therapy versus fixed-field intensity-modulated radiotherapy in radical irradiation for cervical cancer without lymphadenectasis: dosimetric and clinical results. Oncol Res Treat. 2018;41(3):105-109.

13. Sharfo AW, Voet PW, Breedveld S, Mens JW, Hoogeman MS, Heijmen BJ. Comparison of VMAT and IMRT strategies for cervical cancer patients using automated planning. Radiother Oncol. 2015; 114(3):395-401.

14. Jia MX, Zhang X, Yin C, et al. Peripheral dose measurements in cervical cancer radiotherapy: a comparison of volumetric modulated arc therapy and step-and-shoot IMRT techniques. Radiat Oncol. 2014;9:61.

15. Zhai DY, Yin Y, Gong GZ, et al. RapidArc radiotherapy for whole pelvic lymph node in cervical cancer with 6 and $15 \mathrm{MV}$ : a treatment planning comparison with fixed field IMRT. J Radiat Res. 2013;54(1): 166-173.

16. Ren W, Sun C, Lu N, et al. Dosimetric comparison of intensitymodulated radiotherapy and volumetric-modulated arc radiotherapy in patients with prostate cancer: a meta-analysis. J Appl Clin Med Phys. 2016;17(6):254-262.

17. Yoshihiro U, Shingo O, Masaru I, et al. Strategies for reducing ovarian dose in volumetric modulated arc therapy (VMAT) for postoperative uterine cervical cancer. Br J Radiol. 2018;91(1081):20160777.
OncoTargets and Therapy

\section{Publish your work in this journal}

OncoTargets and Therapy is an international, peer-reviewed, open access journal focusing on the pathological basis of all cancers, potential targets for therapy and treatment protocols employed to improve the management of cancer patients. The journal also focuses on the impact of management programs and new therapeutic agents and protocols on

\section{Dovepress}

patient perspectives such as quality of life, adherence and satisfaction. The manuscript management system is completely online and includes a very quick and fair peer-review system, which is all easy to use. Visit http://www.dovepress.com/testimonials.php to read real quotes from published authors. 